\title{
A-D-A Structured Small-Molecule Hole Transporting Materials for Dopant-Free Perovskite Solar Cells
}

\author{
Yuanyuan Kan, Bo Xu, and Ke Gao* \\ Department of Materials Science and Engineering, University of Washington, Seattle, \\ Washington 98195, the United States
}

Email:kegao@uw.edu (K. G.)

\begin{abstract}
Hole-transporting materials (HTMs) are necessary for highly efficient perovskite solar cells (PSCs) to enable the hole carriers extraction and transport. There are designed varieties of HTMs so far, including small molecules and polymers. However, most of them have to be doped to get the high mobility and conductivity. Otherwise, the dopant will deteriorate the long-term stability and efficiency, which is a bottleneck for the commercialization of PSCs. The development for non-doping HTMs, which can replace the traditional doped HTMs, brings great hope to this field. Among them, acceptor (A)-donor (D)-acceptor (A) type non-doping HTMs are an important portion. In this min-review, we summarize all the A-D-A type non-doping HTMs and their properties.
\end{abstract}

Keywords perovskite solar cells, hole transporting materials, non-doping, A-D-A structure

\section{Introduction}

Recently, perovskite solar cells (PSCs) benefiting from their adjustable formulas, strong light absorption, long carrier diffusion length and high carrier mobility have attracted more and more attention from all over the world. ${ }^{[1-6]}$ Generally, the typically used devices of PSCs contain two types of configurations. One is mesoporous fabrication and the other is inversed devices. ${ }^{[7]}$ To date, remarkable progress has been achieved in the past few years after scientists' continuous efforts with power conversion efficiency (PCE) exceeding $23 \%{ }^{[8,9]}$ Besides perovskite absorbing materials, holeransporting materials (HTMs) play crucial roles in extraction and transportation of holes from perovskite to the corresponding electrode. ${ }^{[7]}$ So far, it should be noted that most of PSCs with PCE over $20 \%$ rely mainly on the mesoporous configuration, in which the HTMs are more necessary and important. $^{[10-12]}$

Currently, lots of new HTMs, which include different kinds of inorganic materials and organic materials, have been explored to use as HTMs. ${ }^{[13]}$ However, the most frequently used HTM is still 2,2,7,7-tetrakis( $N, N$-di-p-methoxyphenyl-amine)-9,9-spirobifluorene (Spiro-OMeTAD). ${ }^{[14-16]}$ However, the pristine film of Spiro-OMeTAD has low mobility and conductivity. Therefore, chemical doping via adding some salts is quite necessary for sufficient conductivity to improve the device performance. ${ }^{[17]}$ As we all know, the additives can accelerate degradation of perovskite active layer due to exposure into ambient atmosphere to deliquesce. In addition, the p-type doping strategy complicates the device processing due to a strict optimization of the doping. Thus, it is urgent to develop alternative dopant-free HTMs for high efficient PSCs. ${ }^{[18-20]} A$ large number of dopant-free HTMs have been explored. ${ }^{[20]}$ Among them, acceptor (A)-donor (D)-acceptor (A) type smallmolecule materials are important fractions of HTMs because A-D-A structured materials can facilitate the charge transfer and improve the hole mobility. ${ }^{[21]}$ In the past ten years, hundreds of papers about new type of A-D-A small-molecule HTMs have been designed as functional materials. ${ }^{[2,23]}$ These materials, comparing with traditional HTMs such as Spiro-OMeTAD, feature easy synthesis and tunable energy levels, high mobility and conductivity, low cost. Many reports have been published to further comprehend the relationship between material structure and device. In addition, several reviews have been published to organize and review the non-doping HTMs, but there has not been any review on A-D-A structured small molecules based HTMs. ${ }^{[14,20]}$ Herein, we focus on exhibiting the most effective recent progress in A-D-A type doped-free small-molecule HTMs. Besides, we will discuss for further understanding of structure-properties relationship of the HTMs to the development of this field.
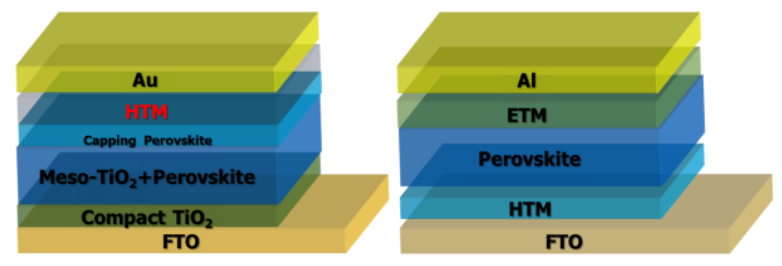

Figure 1 Mesoporous structure ( $n-i-p$, left) and inversed structure (p-i-n, right) of persovekite solar cells.

\section{BDT core based HTMs}

Benzo[1,2-b:4,5-b']dithiophene (BDT) is a famous building unit and has widely used in organic photovoltaics (OPV), in which it serves usually as a donor unit to build polymers or small molecules. There are several advantages for BDT. ${ }^{[2]}$ For one, BDT possesses symmetric and rigid structures, which will facilitate the electron delocalization and improve the $\pi-\pi$ stacking in the films, thus excellent mobility will be expected for BDA-based materials. ${ }^{[25]}$ For the other, the electron donating for BDT is a little weak and thus the resulting materials will exhibit a relatively low highest occupied molecular orbital (HOMO) energy level, which will well match with that of perovskite.

As shown in Figure 2, the cores of all the small molecules with A-D-A structure are all based on BDT. In 2012, Zhou et al. ${ }^{[24]}$ designed and synthesized a small molecule donor 


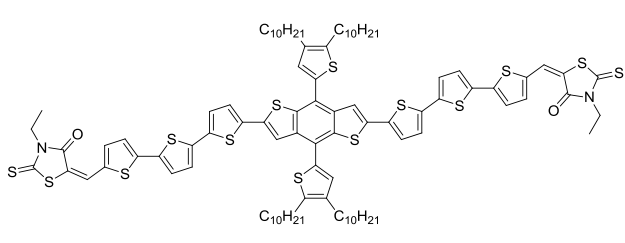

2014-DR3TBDTT

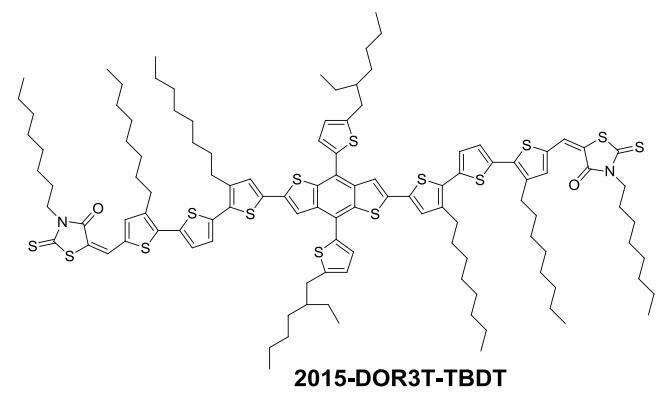

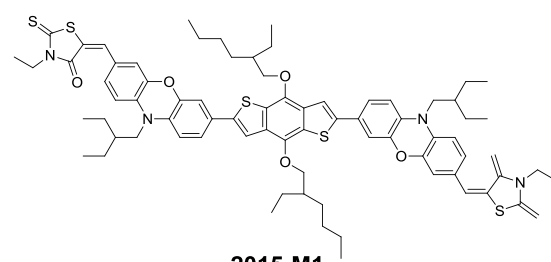

2015-M1

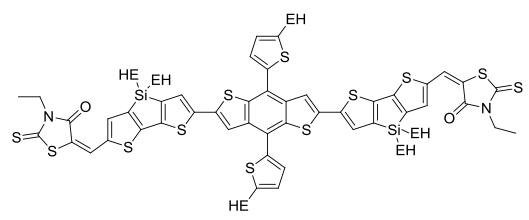

2016-DERDTS-TBDT

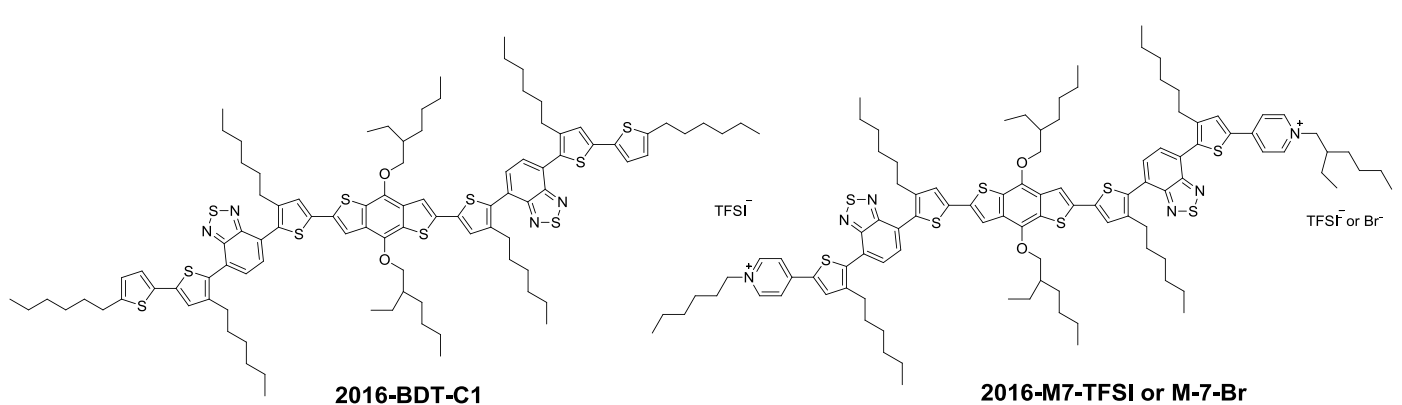

Figure 2 Chemical structures of A-D-A structured non-doping HTMs based on BDT.

called DR3TBDT with BDT as a center and 3-ethylrhodanine as an ending group via the linkage of dioctyltertthiophene. A best PCE of $7.38 \%$ was achieved when DR3TBDT was blended with PCBM to fabricate the organic solar cells, which was the best one for small molecule solar cells then. ${ }^{[24]}$ In 2013 , the same group introduced thiophene at the 4- and 8- positions of BDT for DR3TBDT to extend the conjugation of BDT and 2-ethylhexyl on the thiophene units to increase the solubility, and synthesized DR3TBDTT. ${ }^{[25]}$ A promising PCE of $8.12 \%$ was achieved by blending DR3TBDTT with PCBM and this was also the best value for small molecule solar cells. ${ }^{[25]}$ In 2014, Zheng et al. ${ }^{[26]}$ designed a new and hydrophobic DR3TBDTT by replacing 2-ethylhexyl $(\mathrm{EH})$ with two alkyl groups. The new DR3TBDTT demonstrates a well matched HOMO energy level with that of perovskite. A modest PCE of $8.8 \%$ was achieved when DR3TBDTT was used to perform as non-doping HTL for mesoporous perovskite solar cells, which was comparable to that of PSCs using Siro-OMeOTAD + $\mathrm{Li}+\mathrm{tBP}$. It was also worthy to note that PDMS was used to improve the morphology of DR3TBDTT films. ${ }^{[26]}$ In 2015, Liu et al. ${ }^{[27]}$ used a new DR3TBDT derivative DOR3T-BDT as a non-doping HTL and compared with its properties with those of Siro-OMeOTAD in planar PSCs. Compared with DR3TBDTT, DOR3T-TBDT was designed by introducing more alkys into the tertthiophenes. As shown in Table 1, DR3TBDTT showed a comparable conductivity $\left(4 \times 10^{-4} \mathrm{~S} \cdot \mathrm{cm}^{-1}\right)$ to that of doped Siro-OMeOTAD $\left(\sim 10^{-4} \mathrm{~S} \cdot \mathrm{cm}^{-1}\right)$ and a much bigger mobility $\left(0.26 \mathrm{~cm}^{2} \cdot \mathrm{V}^{-1} \cdot \mathrm{s}^{-1}\right.$, OFET data) than that of spiro-OMeTAD $\left(\sim 10^{-4} \mathrm{~cm}^{2} \cdot \mathrm{V}^{-1} \cdot \mathrm{s}^{-1}\right) \cdot{ }^{[27]}$ The excellent properties for DOR3T-TBDT were translated into a better PCE of $14.9 \%$ than that of doped spiro-OMeTAD because of enhanced $J_{\text {sc }}$. In 2016, the same group designed the other BDT-core molecule DERDTS-TBDT with BDT as core and 3-ethylrodanine as ending groups via the linkage of dithienosilole units, and an excellent PCE of $16.2 \%$ was achieved when the pristine film was used as HTL in planar PSCs. ${ }^{[28]}$ However, non-dopant spiro-OMeTAD based devices demonstrated a poor PCE of $5.4 \%$. The authors ascribe the excellent performance for DERDTS-TBDT to the well matched HOMO energy level and high mobility. ${ }^{[28]}$ A control molecule with deeper HOMO, in which BDT was replaced with 5,6difluoro-2,1,3-benzothiadiazole, was also designed. However, the related devices showed a poor PCE of $6.2 \%$. They argued that the tuning of the energy level for HTMs is of great importance to improve the properties of non-doing HTMs.

In 2015, Cheng et al. ${ }^{[29]}$ designed and synthesized a small molecule M1 with BDT as core and 3-ethylrodanine as end units via the bridge of POZ. In addition, the new material was regarded as low-cost because it can be reached with high yield of $52 \%$ by only 5 steps and the low-cost raw materials are available from the chemical companies. ${ }^{\left[{ }^{[9]}\right.}$ When dopants were not applied at the same time, M1-based PSCs show a much better PCE (13.2\%) than that of Siro-OMeOTAD-based devices. It should be noted that M1-based devices demonstrated a higher $V_{o c}$ than those of Siro-OMeOTAD-based devices because M1 showed a lowed HOMO energy level. When HTMs were doped with dopants, M1-based devices showed a little higher PCE than those of Siro-OMeOTAD-based devices. In addition, for M1 as HTM, the performance of devices are quite similar whether dopants were added or not. The authors also applied M1as small molecule donor into organic solar cells and achieved a moderate PCE of $6.9 \%$. In 2016, the same group reported the other BDT based non-doping small molecule BDT-C1 with BDT as core and benzo-[c][1,2,5]-thiadiazole derivatives (BTz) as ending groups by linking of thiophenes. ${ }^{[30]}$ 
Table 1 Summary of the parameters of A-D-A structured non-doping HTMs and the related devices ${ }^{a}$

\begin{tabular}{|c|c|c|c|c|c|c|c|c|c|}
\hline HTM name & Perovskite & $\mathrm{J}_{\mathrm{sc}} /\left(\mathrm{mA} \cdot \mathrm{cm}^{-2}\right)$ & $V_{\text {oc }} \mathrm{N}$ & $\mathrm{FF} / \%$ & $\sigma /\left(\mathrm{S} \cdot \mathrm{cm}^{-1}\right)$ & $\mu_{\mathrm{h}} /\left(\mathrm{cm}^{2} \cdot \mathrm{V}^{-1} \cdot \mathrm{s}^{-1}\right)$ & $E_{\mathrm{g}} / \mathrm{eV}$ & PCE $/ \%$ & Ref. \\
\hline DR3TBDTT & $\mathrm{CH}_{3} \mathrm{NH}_{3} \mathrm{Pbl}_{3-x} \mathrm{Cl}_{x}$ & 15.3 & 0.95 & 60 & - & - & 1.84 & 8.8 & [26] \\
\hline M1 & $\left(\mathrm{CH}_{3} \mathrm{NH}_{3}\right) \mathrm{Pbl}_{3}$ & 19.1 & 1.016 & 67.8 & $1.16 \times 10^{-3}$ & $2.71 \times 10^{-4}$ & 1.84 & 13.2 & [29] \\
\hline DOR3T-TBDT & $\mathrm{CH}_{3} \mathrm{NH}_{3} \mathrm{Pbl}_{3-x} \mathrm{Cl}_{x}$ & 20.7 & 0.97 & 74 & $4 \times 10^{-4}$ & 0.26 & 1.77 & 14.9 & [27] \\
\hline DERDTS-TBDT & $\mathrm{CH}_{3} \mathrm{NH}_{3} \mathrm{Pbl}_{3-x} \mathrm{Cl}_{x}$ & 21.2 & 1.05 & 72.8 & - & $1.0 \times 10^{-4}$ & 1.83 & 16.2 & [28] \\
\hline BDT-C1 & $\left(\mathrm{CH}_{3} \mathrm{NH}_{3}\right) \mathrm{Pbl}_{3}$ & 18.4 & 1.001 & 75.7 & $1.16 \times 10^{-4}$ & $3.18 \times 10^{-4}$ & 1.74 & 13.4 & [30] \\
\hline M6 & $\left(\mathrm{FAPbl}_{3}\right)_{0.85}\left(\mathrm{MAPbBr}_{3}\right)_{0.15}$ & 19.83 & 1.04 & 65.6 & $1.03 \times 10^{-4}$ & $1.11 \times 10^{-4}$ & 1.84 & 13.5 & [31] \\
\hline M7-TFSI & $\left(\mathrm{FAPbl}_{3}\right)_{0.85}\left(\mathrm{MAPbBr}_{3}\right)_{0.15}$ & 22.84 & 1.095 & 70.6 & $2.01 \times 10^{-4}$ & $3.24 \times 10^{-4}$ & 1.70 & 17.7 & [31] \\
\hline $\mathrm{M} 7-\mathrm{Br}$ & $\left(\mathrm{FAPbl}_{3}\right)_{0.85}\left(\mathrm{MAPbBr}_{3}\right)_{0.15}$ & 21.94 & 1.045 & 67.6 & $1.58 \times 10^{-4}$ & $3.03 \times 10^{-4}$ & 1.70 & 15.5 & [31] \\
\hline POZ2 & $\mathrm{CH}_{3} \mathrm{NH}_{3} \mathrm{Pbl}_{3}$ & 17.8 & 0.971 & 74.2 & $2.01 \times 10^{-4}$ & $5.98 \times 10^{-4}$ & 1.59 & 12.8 & [32] \\
\hline $\mathrm{S}, \mathrm{N}-1$ & $\mathrm{CH}_{3} \mathrm{NH}_{3} \mathrm{Pbl}_{3}$ & 16.6 & 0.953 & 72 & 一 & $4.2 \times 10^{-5}$ & 1.63 & 11.4 & {$[21]$} \\
\hline $\mathrm{S}, \mathrm{N}-2$ & $\mathrm{CH}_{3} \mathrm{NH}_{3} \mathrm{Pbl}_{3}$ & 16.3 & 0.899 & 70 & 一 & $3.5 \times 10^{-5}$ & 1.68 & 10.3 & [21] \\
\hline KR355 & $\left(\mathrm{FAPbl}_{3}\right)_{0.85}\left(\mathrm{MAPbBr}_{3}\right)_{0.15}$ & 16.01 & 1.05 & 53 & - & $5.0 \times 10^{-7}$ & 2.13 & 8.88 & [33] \\
\hline KR321 & $\left(\mathrm{FAPbl}_{3}\right)_{0.85}\left(\mathrm{MAPbBr}_{3}\right)_{0.15}$ & 21.70 & 1.13 & 78 & - & $2.6 \times 10^{-4}$ & 2.05 & 19.03 & [33] \\
\hline KR353 & $\left(\mathrm{FAPbl}_{3}\right)_{0.85}\left(\mathrm{MAPbBr}_{3}\right)_{0.15}$ & 19.31 & 1.12 & 69 & - & $1.1 \times 10^{-5}$ & 1.96 & 14.87 & [33] \\
\hline
\end{tabular}

${ }^{a} \sigma$ : Conductivity; $\mu_{\mathrm{h}}$ : Hole mobility; IP: Ionization potential; $E_{\mathrm{g}}$ : Band gap.

A comparable PCE (13.4\%) was achieved based on pristine BDT-C1 film as HTM compared with doped Siro-OMeOTADbased devices. The excellent performance can be ascribed to appropriate energy levels, high hole mobility and conductivity. When blending BDT-C1 with PCBM in organic solar cells, a moderate PCE of $6.1 \%$ was achieved. ${ }^{\left[{ }^{30]}\right.}$ In the same year, the same group published two A-D-A type ionic molecules as non-doping material M-7-TFSI and M-7-Br. ${ }^{[31]}$ Both molecules are all based on BDT derivate as core and pyridine as ending groups with $\mathrm{BTz}$ derivates as linkages. The only difference between $\mathrm{M}$-7-TFSI and $\mathrm{M}$-7-Br are from the negative ions. The HOMO levels for both molecules are deeper than those of Spiro-OMeOTAD, which will contribute to higher $V_{\text {oc. For }}$ $\mathrm{M}-7-\mathrm{Br}$, the devices showed an average PCE of $15.1 \%$, which is lower than those of M-7-TFSI based devices (17.4\%). M-7-TFSI showed similar performance compared with doped Spiro-OMeOTAD (17.9\%). Similarly, the excellent performance was also ascribed to the suitable energy lever, high mobility and conductivity. Because the energy levels for both molecules are suitable in organic solar cells, the related devices exhibited moderated PCEs of $6.24 \%$ and $6.89 \%$ for $\mathrm{M}-7-\mathrm{Br}$ and $\mathrm{M}-7-\mathrm{TFSI}$ respectively. For the related neural molecule M6 as non-doped HTMs or donor material, PSCs and organic solar cells demonstrated the lowest PCE of $13.0 \%$ and $5.74 \%$, respectively. It should be noted that the PSC in this case used mesoporous configuration and the formulation for perovskite was mixed perovskite $\left(\mathrm{FAPbl}_{3}\right)_{0.85}\left(\mathrm{MAPbBr}_{3}\right)_{0.15}$. As can be seen from Table 1, almost all the BDT-based non-doped HTMs possess the same magnitude order for the hole mobility and conductivity.

\section{Other A-D-A Type HTMs}

In 2015, Cheng et al. ${ }^{[32]}$ designed and synthesized two small molecules POZ2 and POZ3 with phenoxazine as core and dicyanovinyl as ending group via the linkage of $\mathrm{BTz}$ or thiophene, and then applied them into PSCs and organic solar cells. Both of them demonstrate lower HOMO levels than that of perovskite, which can guarantee the hole transport from the perovskite to HTMs. As shown from Table 1, their hole mobilities are quite similar, but the conductivity of POZ2 is much higher than that of POZ3. When both of them were applied as non-dopant HTMs into mesoporous PSCs, the performance for POZ2 (12.8\%) is higher than that for POZ3 (11.5\%). They also applied them as donors in small molecule solar cells, POZ2-based devices demonstrated higher PCE than POZ3-based devices.

In the same year, Grätzel group designed two non-doping HTMs with $S, N$-heteropentacene as central units and DCV as ending units via the linkage of thiophene or ethylenedioxythiophene units. ${ }^{[21]}$ Both of them showed suitable HOMO levels in comparison with that of perovskite, and similar hole mobilities that were lower than the common A-D-A type molecules $\left(\sim 10^{-4}\right.$ $\left.\mathrm{cm}^{2} \cdot \mathrm{V}^{-1} \cdot \mathrm{s}^{-1}\right)$. When applied into mesoporous PSCs, the SN1based devices showed a little better PCE (11.4\%) than that of SN2-based devices (10.3\%). In addition, the IPCE for HTMbased devices was red-shifted compared with the control devices (without HTMs), which demonstrated that the light absorption of HTMs could contributed to the $J_{\text {sc }}$.

In 2017, Nazeeruddin group ${ }^{[33]}$ designed and synthesized three non-doping HTMs with a planar triazatruxene as core and malononitrile as ending units via the linkage of thiophene-based bridges. KR321 based molecule shows the best hole mobility. In addition, KR321 demonstrated an obvious face-on stacking, which facilitates the hole transport. After employment of KR321 as non-doping HTMs for PSCs based on mixed perovskite, the best PCE of $19.03 \%$ was achieved and it has exceeded that for Spiro-OMeTAD (19.01\%). It should be noted that this value is the best result among all the PSCs with A-D-A type non-dopant HTMs.

\section{Conclusions and Perspectives}

In summary, we have reviewed all the non-dopant HTMs based on A-D-A structure. Usually, the A-D-A type HTMs show wide absorption profile, which can be extended to $600-800 \mathrm{~nm}$. More importantly, the A-D-A structured HTMs can facilitate the charge transfer, which can be easier to act as the non-doping 


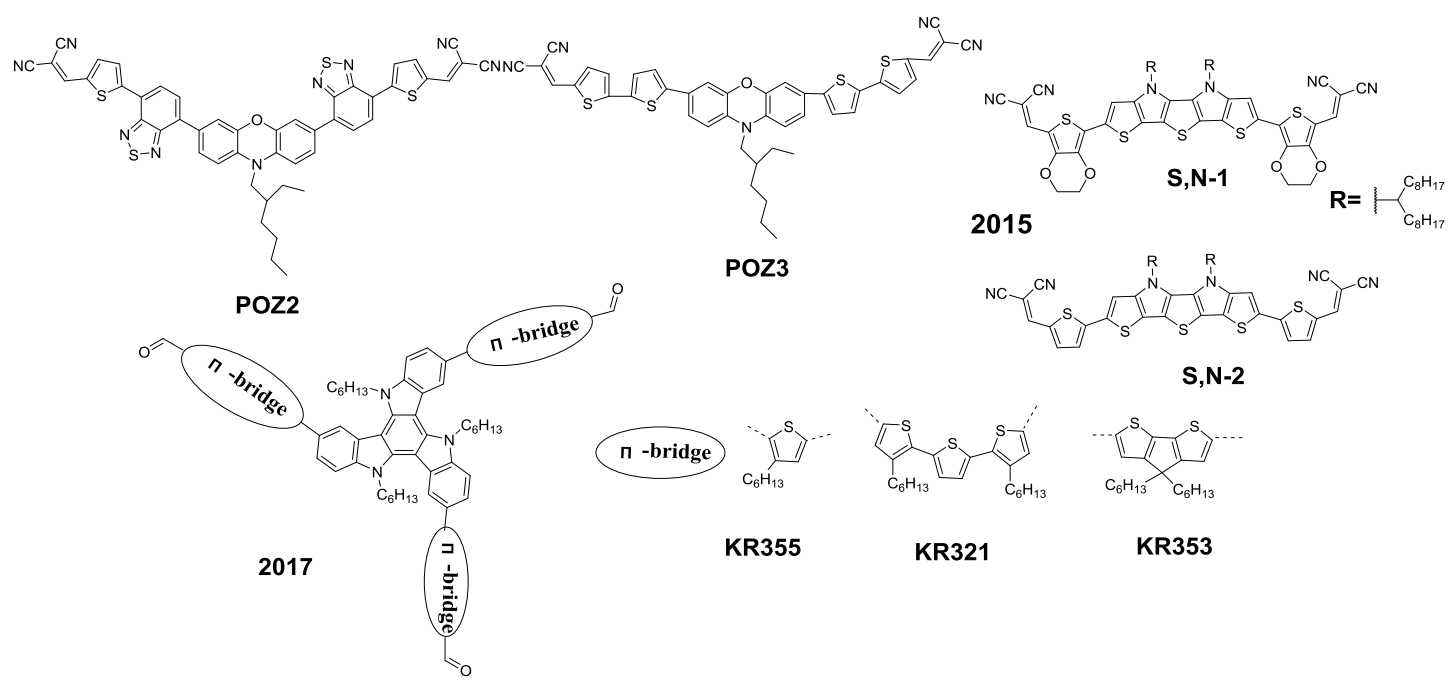

Figure 3 Chemical structures of other A-D-A structured non-doping HTMs.

HTMs compared with other type small molecule HTMs. Suitable energy level matching with the perovskite formulation, hole mobility as well as conductivity are quite important parameters, which can affect the properties of HTMs. In the design of A-D-A structured HTMs, appropriate combinations of donor units and acceptor units should be carefully selected to meet such requirements, although it is very difficult to find some clues liking the chemical structure with the device performance. In fact, most non-dopant HTMs show the hole mobility and conductivity of $10^{-4}$ magnitude order. In addition, the solubility and film-forming property are also necessary factors that should be taken into consideration. So far, the A-D-A non-dopant HTMs-based PSCs have achieved a high PCE of over $19 \%$, which brings much confidence that A-D-A strategy is an efficient way to design the non-dopant HTMs and more efficient and lower cost HTMs with A-D-A structure will be designed for the commercialization of PSCs.

\section{Author Contributions}

Following are the details of the contributions made by each of the authors for the manuscript: Dr. Ke Gao supervised and coordinated the organization of this mini-review. Dr. Ke Gao and Dr. Yuanyuan Kan write the manuscript. Dr. Bo Xu participates in the discussion.

\section{Special Issue}

This paper is dedicated to the special issue of "Organic Optoelectronics \& Photonics" (Guest Editor: Professor Linghai Xie).

\section{References}

[1] Arora, N.; Dar, M. I.; Hinderhofer, A.; Pellet, N.; Schreiber, F.; Zakeeruddin, S. M.; Grätzel, M. Science 2017, eaam5655.

[2] Chen, H.; Ye, F.; Tang, W.; He, J.; Yin, M.; Wang, Y.; Xie, F.; Bi, E.; Yang, X.; Grätzel, M. Nature 2017, 550, 92.

[3] Luo, D.; Yang, W.; Wang, Z.; Sadhanala, A.; Hu, Q.; Su, R.; Shivanna, R.; Trindade, G. F.; Watts, J. F.; Xu, Z. Science 2018, 360, 1442.

[4] Rong, Y.; Hu, Y.; Mei, A.; Tan, H.; Saidaminov, M. I.; Seok, S. I.; McGehee, M. D.; Sargent, E. H.; Han, H. Science 2018, 361, eaat8235

[5] Zhou, H.; Chen, Q.; Li, G.; Luo, S.; Song, T.-b.; Duan, H.-S.; Hong, Z.; You, J.; Liu, Y.; Yang, Y. Science 2014, 345, 542.
[6] Gao, K.; Zhu, Z.; Xu, B.; Jo, S. B.; Kan, Y.; Peng, X.; Jen, A. K. Y. Adv. Mater. 2017, 29, 1703980.

[7] Lee, M. M.; Teuscher, J.; Miyasaka, T.; Murakami, T. N.; Snaith, H. J. Science 2012, 1228604.

[8] Saidaminov, M. I.; Kim, J.; Jain, A.; Quintero-Bermudez, R.; Tan, H.; Long, G.; Tan, F.; Johnston, A.; Zhao, Y.; Voznyy, O. Nat. Energy 2018, 3, 648.

[9] Rajagopal, A.; Yao, K.; Jen, A. K. Adv. Mater. 2018, 30, e1800455.

[10] Bi, D.; Xu, B.; Gao, P.; Sun, L.; Graetzel, M.; Hagfeldt, A. Nano Energy 2016, 23, 138.

[11] Jeon, N. J.; Na, H.; Jung, E. H.; Yang, T.-Y.; Lee, Y. G.; Kim, G; Shin, H.-W.; Seok, S. I.; Lee, J.; Seo, J. Nat. Energy 2018, 3, 682.

[12] Wang, Q.; Chen, B.; Liu, Y.; Deng, Y.; Bai, Y.; Dong, Q.; Huang, J. Energ. Environ. Sci. 2017, 10, 516.

[13] Gao, K.; Xu, B.; Hong, C.; Shi, X.; Liu, H.; Li, X.; Xie, L.; Jen, A. K. Y. Adv. Energy Mater. 2018, 1800809.

[14] Calió, L.; Kazim, S.; Grätzel, M.; Ahmad, S. Angew. Chem. Int. Ed. 2016, 55, 14522.

[15] Correa-Baena, J.-P.; Abate, A.; Saliba, M.; Tress, W.; Jacobsson, T. J.; Grätzel, M.; Hagfeldt, A. Energ. Environ. Sci. 2017, 10, 710.

[16] Dong, H.; Wu, Z.; Xi, J.; Xu, X.; Zuo, L.; Lei, T.; Zhao, X.; Zhang, L.; Hou, X.; Jen, A. K. Y. Adv. Funct. Mater. 2018, 28, 1870013.

[17] Jeon, N. J.; Lee, H. G.; Kim, Y. C.; Seo, J.; Noh, J. H.; Lee, J.; Seok, S. I. J. Am. Chem. Soc. 2014, 136, 7837.

[18] Zhang, H.; Wang, H.; Chen, W.; Jen, A. K. Y. Adv. Mater. 2017, 29, 1604984.

[19] Xu, Y.; Bu, T.; Li, M.; Qin, T.; Yin, C.; Wang, N.; Li, R.; Zhong, J.; Li, H.; Peng, Y. ChemSusChem 2017, 10, 2578.

[20] Zhou, W.; Wen, Z.; Gao, P. Adv. Energy Mater. 2018, 8, 1702512.

[21] Steck, C.; Franckevičius, M.; Zakeeruddin, S. M.; Mishra, A.; Bäuerle, P.; Grätzel, M. J. Mater. Chem. A 2015, 3, 17738.

[22] Rodríguez-Seco, C.; Cabau, L.; Vidal-Ferran, A.; Palomares, E. Acc. Chem. Res. 2018, 51, 869.

[23] Kim, G.-W.; Kang, G.; Kim, J.; Lee, G.-Y.; Kim, H. I.; Pyeon, L.; Lee, J.; Park, T. Energ. Environ. Sci. 2016, 9, 2326.

[24] Zhou, J.; Wan, X.; Liu, Y.; Zuo, Y.; Li, Z.; He, G.; Long, G.; Ni, W.; Li, C.; Su, X.; Chen, Y. J. Am. Chem. Soc. 2012, 134, 16345.

[25] Zhou, J.; Zuo, Y.; Wan, X.; Long, G.; Zhang, Q.; Ni, W.; Liu, Y.; Li, Z.; He, G.; Li, C.; Kan, B.; Li, M.; Chen, Y. J. Am. Chem. Soc. 2013, 135, 8484 .

[26] Zheng, L.; Chung, Y. H.; Ma, Y.; Zhang, L.; Xiao, L.; Chen, Z.; Wang, S.; Qu, B.; Gong, Q. Chem. Commun. 2014, 50, 11196.

[27] Liu, Y.; Chen, Q.; Duan, H.-S.; Zhou, H.; Yang, Y.; Chen, H.; Luo, S.; Song, T.-B.; Dou, L.; Hong, Z.; Yang, Y. J. Mater. Chem. A 2015, 
3, 11940.

[28] Liu, Y.; Hong, Z.; Chen, Q.; Chen, H.; Chang, W. H.; Yang, Y. M.; Song, T. B.; Yang, Y. Adv. Mater. 2016, 28, 440.

[29] Cheng, M.; Xu, B.; Chen, C.; Yang, X.; Zhang, F.; Tan, Q.; Hua, Y.; Kloo, L.; Sun, L. Adv. Energy Mater. 2015, 5, 1401720.

[30] Cheng, M.; Aitola, K.; Chen, C.; Zhang, F.; Liu, P.; Sveinbjörnsson, K.; Hua, Y.; Kloo, L.; Boschloo, G.; Sun, L. Nano Energy 2016, 30, 387.

[31] Chen, C.; Cheng, M.; Liu, P.; Gao, J.; Kloo, L.; Sun, L. Nano Energy 2016, 23, 40.
[32] Cheng, M.; Chen, C.; Yang, X.; Huang, J.; Zhang, F.; Xu, B.; Sun, L. Chem. Mater. 2015, 27, 1808.

[33] Rakstys, K.; Paek, S.; Gao, P.; Gratia, P.; Marszalek, T.; Grancini, G.; Cho, K. T.; Genevicius, K.; Jankauskas, V.; Pisula, W.; Nazeeruddin, M. K. J. Mater. Chem. A 2017, 5, 7811.

Received October 11, 2018 Accepted November 21, 2018 